\title{
SECOND-ORDER SOLUTION OF A NONLINEAR WAVE EQUATION
}

\author{
BY \\ R. W. LARDNER* \\ University of Cape Town
}

\begin{abstract}
The method of multiple scales is used to investigate the nonlinear wave equation $u_{t t}-u_{x x}=\varepsilon\left(2 u_{x} u_{x x}-2 \lambda u_{t}\right)$.

The purpose of the investigation is to find the second-order solution, and in particular to find an approximate solution which may be used for values of $t$ which are of order $\varepsilon^{-2}$.
\end{abstract}

1. Introduction. We shall investigate the nonlinear wave equation

$$
u_{t t}-u_{x x}=\varepsilon\left(2 u_{x} u_{x x}-2 \lambda u_{t}\right)
$$

where $\varepsilon \ll 1$ is a small parameter. This equation has application to the plane motions of a nonlinear elastic rod or column in the case when the damping is viscous in nature and when both the nonlinearity and damping effects are small.

Previous investigations of this equation have used either a modified KrylovBogoliubov method [1] or [2] or the direct two-variable expansion method of Chikwendu and Kevorkian [3]. In both cases, only the lowest-order solution was obtained, that is, a solution which remains valid up to values of $t$ which are $O\left(\varepsilon^{-1}\right)$. Our purpose in the present paper will be to extend this solution to the next order which will allow us to consider values of $t$ as large as $O\left(\varepsilon^{-2}\right)$.

The method of approach will be a three-variable expansion method [4]. We introduce two slow times $\tau=\varepsilon t, \sigma=\varepsilon^{2} t$ and seek the solution in the form

$$
u=u_{0}(x, t, \tau, \sigma)+\varepsilon u_{1}(x, t, \tau, \sigma)+\varepsilon^{2} u_{2}(x, t, \tau, \sigma)+\cdots
$$

The $\tau$ - and $\sigma$-dependences are determined by systematically removing the secular terms from $u_{1}, u_{2}$, etc.

Having found $u_{0}$ and $u_{1}$, we can take the approximation

$$
u \approx u_{0}(x, t, \tau, \sigma)+\varepsilon u_{1}(x, t, \tau, 0)
$$

which for $t=O\left(\varepsilon^{-1}\right)$ has an error which is $O(\varepsilon)$. Thus we are able to refine the accuracy of the previously determined solution for times of order $\varepsilon^{-1}$. However, the more interesting approximation is simply

$$
u \approx u_{0}(x, t, \tau, \sigma)
$$

which is accurate to within an error of $o(1)$ for $t=O\left(\varepsilon^{-2}\right)$.

* Received February 20, 1980.

* Permanent address: Mathematics Department, Simon Fraser University, Burnaby, BC, Canada. 
The lowest-order solution $u_{0}$ is found, as usual, to consist of a superposition of two traveling waves with slowly modulating amplitudes. The $\tau$-dependence of the two amplitudes, which describes the modulation on a time scale of order $\varepsilon^{-1}$, is described by a familiar first-order differential equation whose solution is explicitly known. This solution may involve shock waves, but if $\lambda$ is greater than a certain lower bound shocks do not occur and in this case we can proceed to the longer time scale. It is found that the $\sigma$-dependence of the wave amplitude is obtained as the solution of a telegraph equation. The initial data (i.e. for $\sigma=0$ ) to be used in conjunction with this telegraph equation are obtained from the limiting values of the first-order solution as $\tau \rightarrow \infty$.

In the final section of the paper the case in which the solution involves shocks is discussed. It is shown that the solution without shocks can be extended to this case if Eq. (1) is supplemented with a suitable jump condition. The shock speed is derived for the simplest such condition.

2. The first-order solution. Substituting the form (2) for $u$ into equation (1) in which we make the change $\partial / \partial t \rightarrow \partial / \partial t+\varepsilon(\partial / \partial \tau)+\varepsilon^{2}(\partial / \partial \sigma)$, then comparing the coefficients of the various powers of $\varepsilon$, we obtain the following hierarchy of equations for $u_{0}, u_{1}$, and $u_{2}$ :

$$
\begin{aligned}
u_{0 t t}-u_{0 x x}= & 0 \\
u_{1 t t}-u_{1 x x}= & -2 u_{0 t \tau}+2 u_{0 x} u_{0 x x}-2 \lambda u_{0 t}, \\
u_{2 t t}-u_{2 x x}= & -2 u_{1 t \tau}-u_{0 \tau \tau}-2 u_{0 t \sigma}+2 u_{1 x} u_{0 x x} \\
& +2 u_{0 x} u_{1 x x}-2 \lambda u_{0 \tau}-2 \lambda u_{1 t} .
\end{aligned}
$$

The solution of the first of these equations has the form

$$
u_{0}=G(\alpha, \tau, \sigma)-H(\beta, \tau, \sigma)
$$

where $\alpha=t+x$ and $\beta=t-x$. $G$ and $H$ are general functions of the indicated variables which represent respectively waves traveling in the negative and positive $x$-directions. The $\tau$ - and $\sigma$-dependences of these functions provide a slow modulation of the wave amplitudes.

Substituting (6) into Eq. (4) and changing to $\alpha, \beta$ as variables, we obtain

$$
4 u_{1 \times \beta}=-2 G_{x \tau}+2 H_{\beta \tau}+2\left(G_{x}+H_{\beta}\right)\left(G_{x \chi}-H_{\beta \beta}\right)-2 \lambda\left(G_{x}-H_{\beta}\right) .
$$

After integration this becomes

$$
\begin{aligned}
4 u_{1}= & \beta\left(-2 G_{\tau}+G_{x}^{2}-2 \lambda G\right)+\alpha\left(2 H_{\tau}-H_{\beta}^{2}+2 \lambda H\right)+2\left(G_{x} H-G H_{\beta}\right) \\
& +4\left[G_{1}(\alpha, \tau, \sigma)-H_{1}(\beta, \tau, \sigma)\right]
\end{aligned}
$$

where the last terms arise as the complementary function. Clearly the terms growing linearly with $\alpha$ and $\beta$ are secular and must be eliminated if we are to have a uniformly valid expansion. Thus we obtain the conditions

$$
G_{\tau}=\frac{1}{2} G_{x}^{2}-\lambda G, \quad H_{\tau}=\frac{1}{2} H_{\beta}^{2}-\lambda H
$$

and in addition we are left with:

$$
u_{1}=\frac{1}{2}\left[G_{x}(\alpha, \tau, \sigma) H(\beta, \tau, \sigma)-G(\alpha, \tau, \sigma) H_{\beta}(\beta, \tau, \sigma)\right]+G_{1}(\alpha, \tau, \sigma)-H_{1}(\beta, \tau, \sigma) .
$$


(It is noteworthy that while $u_{0}$ consists of the superposition of the two traveling waves $G$ and $-H, u_{1}$ does not consist of such a superposition, but contains also a nonlinear interference term.)

In order to solve Eq. (7) we differentiate with respect to $\alpha$ or $\beta$ as appropriate and introduce $g=G_{x}, h=H_{\beta}$, obtaining:

$$
g_{\tau}=g g_{x}-\lambda g, \quad h_{\tau}=h h_{\beta}-\lambda h .
$$

These equations may then readily be solved by Lagrange's method, the solutions being

$$
\begin{aligned}
& g(\alpha, \tau, \sigma)=e^{-\lambda \tau} \phi\left(\alpha_{1}, \sigma\right), \quad \alpha_{1}=\alpha+\tau_{1} \phi\left(\alpha_{1}, \sigma\right) \\
& h(\beta, \tau, \sigma)=e^{-\lambda \tau} \psi\left(\beta_{1}, \sigma\right), \quad \beta_{1}=\beta+\tau_{1} \psi\left(\beta_{1}, \sigma\right)
\end{aligned}
$$

where $\tau_{1}=\lambda^{-1}\left(1-e^{-\lambda \tau}\right)$. The solution obtained in previous investigations $[1,2]$ is obtained from (9) by setting $\sigma \equiv 0$.

In terms of $g$ and $h$, we have

$$
u_{0 x}=g(\alpha, \tau, \sigma)+h(\beta, \tau, \sigma), \quad u_{0 t}=g(\alpha, \tau, \sigma)-h(\beta, \tau, \sigma) .
$$

Setting $t=\tau=\sigma=0$ in these, we obtain that

$$
g(x, 0,0)=\frac{1}{2}\left[u_{0 x}(x, 0)+u_{0 t}(x, 0)\right], \quad h(-x, 0,0)=\frac{1}{2}\left[u_{0 x}(x, 0)-u_{0 t}(x, 0)\right] .
$$

Thus the initial values $g(\alpha, 0,0)$ and $h(\beta, 0,0)$ are determined for all values of $\alpha$ and $\beta$ respectively from the initial values of $u_{0 x}$ and $u_{0 t}$.

Setting $\tau=\sigma=0$ in Eq. (9), we obtain that $\alpha_{1}=\alpha$ and $\beta_{1}=\beta$, and therefore

$$
\phi\left(\alpha_{1}, 0\right)=g\left(\alpha_{1}, 0,0\right), \quad \psi\left(\beta_{1}, 0\right)=h\left(\beta_{1}, 0,0\right) .
$$

Thus $\phi\left(\alpha_{1}, 0\right)$ and $\psi\left(\beta_{1}, 0\right)$ are known for all $\alpha_{1}, \beta_{1}$. Eqs. (9) then determine the complete solution $g(\alpha, \tau, 0)$ and $h(\beta, \tau, 0)$. The lines $a_{1}=$ const. in the $\alpha \tau$-plane are charqcteristics for $g$ along which $g(\alpha, \tau, 0)$ decays exponentially from its initial value $\phi\left(\alpha_{1}, 0\right)$, and similarly $h$ decays exponentially along the characteristics $\beta_{1}=$ const.

This solution remains valid for all $(\alpha, \tau)$ or $(\beta, \tau)$ as long as the two families of characteristics have no envelopes in $\tau>0$. In general, however, the solution for $g(\alpha, \tau, 0)$ is valid only for $\tau \leq \tau_{g}$ where

$$
\lambda^{-1}\left(1-e^{-\lambda \tau_{g}}\right)=\left[\phi^{\prime}\left(\alpha_{m}, 0\right)\right]^{-1},
$$

where $\alpha_{m}$ is the value of $\alpha_{1}$ at which $\phi^{\prime}\left(\alpha_{1}, 0\right)$ has its largest positive value. For $\tau>\tau_{g}$ a shock wave occurs in the solution. However, if $\lambda \geq \phi^{\prime}\left(\alpha_{m}, 0\right)$, no real solution for $\tau_{g}$ exists so that in this case the lowest-order solution $g(\alpha, \tau, 0)$ given by $(9)$ remains valid for all $\tau$ and no shock occurs. Similarly, if $\lambda \geq \psi^{\prime}\left(\beta_{m}, 0\right)$ no shock occurs in $h(\beta, \tau, 0)$. We shall assume in the following that these conditions are met. The inclusion of shocks will be discussed in the final section.

In calculating the next approximation, it turns out to be more convenient to take the first-order solution in the following slightly different form:

$$
g(\alpha, \tau, \sigma)=e^{-\lambda \tau} \Phi(A, \sigma), \quad h(\beta, \tau, \sigma)=e^{-\lambda \tau} \Psi(B, \sigma)
$$

where

$$
A=\alpha-\lambda^{-1} e^{-\lambda t} \Phi(B, \sigma), \quad B=\beta-\lambda^{-1} e^{-\lambda \tau} \Psi(B, \sigma)
$$


$A$ and $B$ are simply different characteristic coordinates from the previous $\alpha_{1}, \beta_{1}$, for example $A=\alpha_{1}-\lambda^{-1} \phi\left(\alpha_{1}, \sigma\right)$. When $\sigma=0$, the initial values $\Phi(A, 0)$ are determined implicitly from the conditions

$$
\Phi(A, 0)=g(\alpha, 0,0), \text { where } A=\alpha-\lambda^{-1} g(\alpha, 0,0) .
$$

This determination is unique provided

$$
d A / d \alpha=1-\lambda^{-1} g^{\prime}(\alpha, 0,0) \geq 0
$$

for all $\alpha$, and this is precisely the condition that no shocks occur. Thus the solution in the form (13), (14) can be used for $\sigma=0$ if and only if shocks are absent.

The significance of the characteristic coordinate $A$ is that it represents the asymptotic value of $\alpha$ on the characteristic as $\tau \rightarrow \infty$. In contrast, $\alpha_{1}$ represents the initial value of $\alpha$ at $\tau=0$ (see Fig. 1). In particular, it follows that

$$
\Phi(A, 0)=\lim _{\tau \rightarrow \infty} e^{\lambda \tau} g(A, \tau, 0)
$$

with a similar expression for $\Psi(B, 0)$. Thus the initial values of $\Phi$ and $\Psi$ for $\sigma=0$ are determined from the asymptotic values of the first-order solution $g(\alpha, \tau, 0), h(\beta, \tau, 0)$ as $\tau \rightarrow \infty$.

3. The second-order solution. We now substitute the expressions (6) and (8) into Eq. (5). After some simplification we obtain

$$
\begin{aligned}
4 u_{2 x \beta}= & -2 G_{1 x \tau}-G_{\tau \tau}-2 G_{x \sigma}+2 G_{x x} G_{1 x}+2 G_{x} G_{1 x x}-2 \lambda G_{\tau}-2 \lambda G_{1 x} \\
& +2 H_{1 \beta \tau}+H_{\tau \tau}+2 H_{\beta \sigma}-2 H_{\beta \beta} H_{1 \beta}-2 H_{\beta} H_{1 \beta \beta}+2 \lambda H_{\tau}+2 \lambda H_{1 \beta} \\
& +G_{\tau} H_{\beta \beta}-G_{x x} H_{\tau}+2 G_{x}^{2} H_{\beta \beta}-2 G_{x x} H_{\beta}^{2} \\
& +\left\{\left(G G_{x}\right)_{x} H_{\beta \beta}-\left(H H_{\beta}\right)_{\beta} G_{x x}-5 G_{x} G_{x x} H_{\beta}+5 G_{x} H_{\beta} H_{\beta \beta}\right. \\
& +2 G_{x x} H_{1 \beta}-2 H_{\beta \beta} G_{1 x}-2 G_{x} H_{1 \beta \beta}+2 G_{1 x x} H_{\beta} \\
& \left.-G G_{x} H_{\beta \beta \beta}+H H_{\beta} G_{x x x}\right\} .
\end{aligned}
$$

The last group of terms bracketed together produce non-secular terms when integrated, and therefore do not need to be retained explicitly to this order. After integration, therefore, we get:

$$
\begin{aligned}
4 u_{2}= & \beta\left\lfloor-2 G_{1 \tau}-2 G_{\sigma}+2 G_{\chi} G_{1 x}-2 \lambda G_{1}-\int\left(G_{\tau \tau}+2 \lambda G_{\tau}\right) d \alpha\right\rfloor \\
& +\alpha\left\lfloor 2 H_{1 \tau}+2 H_{\sigma}-2 H_{\beta} H_{1 \beta}+2 \lambda H_{1}+\int\left(H_{\tau \tau}+2 \lambda H_{\tau}\right) d \beta\right\rfloor \\
& +H_{\beta} \int\left(G_{\tau}+2 G_{\chi}^{2}\right) d \alpha-G_{\chi} \int\left(H_{\tau}+2 H_{\beta}^{2}\right) d \beta+N S T .
\end{aligned}
$$

The integral terms here are secular in the sense that in general they produce terms which grow linearly with $\alpha$ or $\beta$. If $P(\alpha)$ is any bounded function of $\alpha$, we define

$$
\bar{P}=\lim _{x \rightarrow \infty} \frac{1}{\alpha} \int P(\alpha) d \alpha .
$$




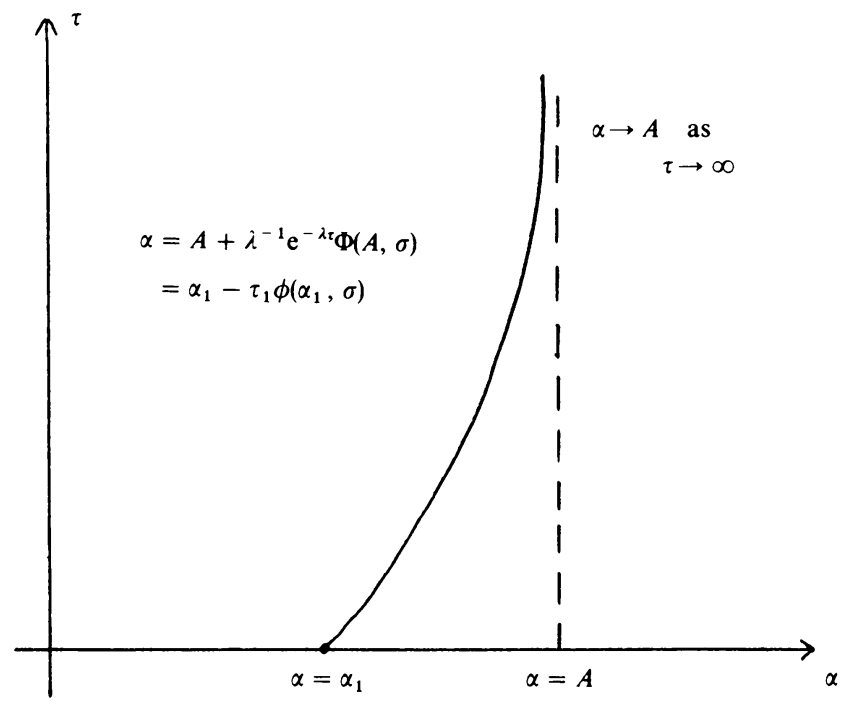

Fig. 1.

Then we can write

$$
\int P(\alpha) d \alpha=\bar{P} \alpha+R(\alpha)
$$

where the remainder $R(\alpha)$ is non-secular, i.e. $\alpha^{-1} R(\alpha) \rightarrow 0$ as $\alpha \rightarrow \infty$. Making this construction for each of the integrals in (16) we find that the leading secular terms are proportional to $\alpha \beta$. Setting these to zero we get the condition

$$
\bar{G}_{\tau \tau}+2 \lambda \bar{G}_{\tau}=\bar{H}_{\tau \tau}+2 \lambda \bar{H}_{\tau} .
$$

In addition, there are also secular terms growing linearly with $\alpha$ or $\beta$, and setting these to zero we get:

$$
\begin{gathered}
G_{1 \tau}-G_{x} G_{1 \alpha}+\lambda G_{1}=-G_{\sigma}-\int\left(\frac{1}{2} G_{\tau \tau}+\lambda G_{\tau}\right) d \alpha+\alpha\left(\frac{1}{2} \bar{G}_{\tau \tau}+\lambda \bar{G}_{\tau}\right)-G_{x}\left(\frac{1}{2} \bar{H}_{\tau}+\overline{H_{\beta}^{2}}\right) \\
H_{1 \tau}-H_{\beta} H_{1 \beta}+\lambda H_{1}=-H_{\sigma}-\int\left(\frac{1}{2} H_{\tau \tau}+\lambda H_{\tau}\right) d \beta+\beta\left(\frac{1}{2} \bar{H}_{\tau \tau}+\lambda \bar{H}_{\tau}\right)-H_{\beta}\left(\bar{G}_{\tau}+\overline{G_{\chi}^{2}}\right) .
\end{gathered}
$$

In order to solve Eqs. (18) and (19) for $G_{1}$ and $H_{1}$ we substitute from Eqs. (13), (14) for the first-order functions. From these latter equations, after integration, we have that

where

$$
G(\alpha, \tau, \sigma)=e^{-\lambda \tau} \Phi_{1}(A, \sigma)+\frac{1}{2} \lambda^{-1} e^{-2 \lambda \tau}[\Phi(A, \sigma)]^{2}
$$

$$
\Phi_{1}(A, \sigma) \equiv \int \Phi(A, \sigma) d A
$$

with a similar expression for $H(\beta, \tau, \sigma)$. It is convenient to use $A$ and $B$ as variables in place of $\alpha$ and $\beta$ in Eqs. (18) and (19), in which case it is readily seen that

$$
G_{1 \tau}-G_{\chi} G_{1 x}=\left.\frac{\partial G_{1}}{\partial \tau}\right|_{A}, \quad H_{1 \tau}-H_{\beta} H_{1 \beta}=\left.\frac{\partial H_{1}}{\partial \tau}\right|_{B} .
$$


We also obtain from $(20)$ that

$$
\begin{aligned}
& G_{\sigma}=e^{-\lambda \tau} \frac{\partial \Phi_{1}}{\partial \sigma}, \quad G_{\tau}=-\lambda e^{-\lambda \tau} \Phi_{1}, \\
& G_{\tau \tau}=\lambda^{2} e^{-\lambda \tau} \Phi_{1}-\lambda e^{-2 \lambda \tau} \Phi^{2}\left[1+\lambda^{-1} e^{-\lambda \tau} \Phi_{A}\right]^{-1},
\end{aligned}
$$

where

$$
\Phi_{A} \equiv \partial \Phi / \partial A
$$

Hence we get

$$
\begin{aligned}
& \int G d \alpha=e^{-\lambda \tau} \mid \int \Phi_{1} d A-\frac{1}{2} \lambda^{-1} e^{-\lambda \tau} \int \Phi^{2} d A+\lambda^{-1} e^{-\lambda \tau} \Phi_{1} \Phi_{A}+\frac{1}{6} \lambda^{-2} e^{-2 \lambda \tau} \Phi^{3} ! \\
& \int G_{\tau} d \alpha=e^{-\lambda \tau} \mid-\lambda \int \Phi_{1} d A+e^{-\lambda \tau} \int \Phi^{2} d A-e^{-\lambda \tau} \Phi_{1} \Phi_{\mid}, \\
& \int G_{\tau \tau} d \alpha=e^{-\lambda \tau} \mid \lambda^{2} \int \Phi_{1} d A-2 \lambda e^{-\lambda \tau} \int \Phi^{2} d A+\lambda e^{-\lambda \tau} \Phi_{1} \Phi !
\end{aligned}
$$

Since we require that $G$ should be bounded for large $\alpha$, then $\Phi$ and $\Phi_{1}$ must both be bounded for large $A$. So, from (14), $A \sim \alpha$ as $\alpha \rightarrow \infty$. Therefore from (23)-(25) we obtain that

$$
\begin{aligned}
\bar{G} & =e^{-\lambda \tau}\left(\overline{\Phi_{1}}-\frac{1}{2} \lambda^{-1} \cdot e^{-\lambda \tau} \overline{\Phi^{2}}\right), \quad \bar{G}_{\tau}=e^{-\lambda \tau}\left(-\lambda \overline{\Phi_{1}}+e^{-\lambda \tau} \overline{\Phi^{2}}\right), \\
\bar{G}_{\tau \tau} & =e^{-\lambda \tau}\left(\lambda^{2} \overline{\Phi_{1}}-2 \lambda e^{-\lambda \tau} \overline{\Phi^{2}}\right),
\end{aligned}
$$

where

$$
\overline{\Phi_{1}}=\lim _{A \rightarrow \infty} \frac{1}{A} \int \Phi_{1} d A, \quad \overline{\Phi^{2}}=\lim _{A \rightarrow \infty} \frac{1}{A} \int \Phi^{2} d A .
$$

A corresponding set of results to (22)-(26) is obtained also for $H$. Substituting from (26) and the corresponding equations for $H$ into the condition (17) we obtain that $\bar{\Phi}_{1}=\bar{\Psi}_{1}$. From its definition (21), $\Phi_{1}$ contains an arbitrary function of $\sigma$ and so does $\Psi_{1}$, so that this condition establishes a relation between these two arbitrary functions.

Substituting for $G$ and $H$ into Eq. (18), we get

$$
\begin{aligned}
\frac{\partial G_{1}}{\partial \tau}+\lambda G_{1}= & e^{-\lambda \tau}\left[-\frac{\partial \Phi_{1}}{\partial \sigma}+\frac{1}{2} \lambda\left(\lambda \int \Phi_{1} d A+e^{-\lambda \tau} \Phi_{1} \Phi\right)-\frac{1}{2} \lambda^{2} \bar{\Phi}_{1} \alpha\right. \\
& -\frac{1}{2} \Phi e^{-\lambda \tau}\left(-\lambda \bar{\Psi}_{1}+3 e^{-\lambda \tau} \overline{\Psi^{2}}\right) \mid
\end{aligned}
$$

after using the fact that $\overline{H_{\beta}^{2}}=2 \bar{H}_{\tau}+2 \lambda \bar{H}$ which follows from (7). That is,

$$
\frac{\partial}{\partial \tau}\left(e^{\lambda \tau} G_{1}\right)=\left[-\frac{\partial \Phi_{1}}{\partial \sigma}+\frac{1}{2} \lambda^{2} \int \Phi_{1} d A-\frac{1}{2} \lambda^{2} \bar{\Phi}_{1} A\right]+\frac{1}{2} \lambda \Phi_{1} \Phi e^{-\lambda \tau}-\frac{3}{2} \Phi \overline{\Psi^{2}} e^{-\lambda \tau} .
$$

Thus, after integrating we get:

$G_{1}=\left[-\frac{\partial \Phi_{1}}{\partial \sigma}+\frac{1}{2} \lambda^{2} \int\left(\Phi_{1}-\overline{\Phi_{1}}\right) d A\right] \tau e^{-\lambda \tau}-\frac{1}{2} \Phi_{1} \Phi e^{-2 \lambda \tau}+\frac{3}{4 \lambda} \Phi \overline{\Psi^{2}} e^{-3 \lambda \tau}+\tilde{\Phi}(A, \sigma) e^{-\lambda \tau}$,

the last term being the "constant" of integration. 
The first term in this expression is secular since, if it is present then for large $\tau$ we have

$$
\varepsilon G_{1} / G \sim \text { const. } \varepsilon \tau
$$

which becomes $O(1)$ when $t=O\left(\varepsilon^{-2}\right)$. To preserve uniformity of the expansion for $t$ of order $\varepsilon^{-2}$ we must therefore set the coefficient of this term equal to zero:

$$
\frac{\partial \Phi_{1}}{\partial \sigma}=\frac{1}{2} \lambda \int\left(\Phi_{1}-\bar{\Phi}_{1}\right) d A .
$$

A similar condition holds for $\Psi$. After differentiating with respect to $A$ or $B$ respectively, these conditions take the form of (modified) telegraph equations

$$
\partial^{2} \Phi_{1} / \partial A \partial \sigma=\frac{1}{2} \lambda^{2}\left(\Phi_{1}-\bar{\Phi}_{1}\right), \quad \partial^{2} \Psi_{1} / \partial B \partial \sigma=\frac{1}{2} \lambda^{2}\left(\Psi_{1}-\Psi_{1}\right) .
$$

A further differentiation produces pure telegraph equations for the functions $\Phi$ and $\Psi$ :

$$
\partial^{2} \Phi / \partial A \partial \sigma=\frac{1}{2} \lambda^{2} \Phi, \quad \partial^{2} \Psi / \partial B \partial \sigma=\frac{1}{2} \lambda^{2} \Psi .
$$

4. Discussion; remarks on shock fitting. The first-order solution of the nonlinear wave equation (1) is given by (6) where in lowest order $g=G_{x}$ and $h=H_{\beta}$ are most conveniently taken in the form (9). When $\sigma=0$, the functions $\phi\left(\alpha_{1}, 0\right)$ and $\psi\left(\beta_{1}, 0\right)$ are given explicitly in terms of the initial data by (11) and (12) and then Eqs. (9) allow the solution $g(\alpha, \tau, 0)$ and $h(\beta, \tau, 0)$ to be readily constructed.

When constructing the next-order solution (i.e. $\sigma \neq 0$ ), however, it is more convenient to take the solution in the form (13), (14). This is permissible if and only if the lowestorder solution has no shocks, which means that the damping coefficient $\lambda$ must be greater than some lower bound which depends on the initial data. The initial values $\Phi(A, 0)$ are determined implicitly from the initial data by (15) and (11) and similarly for $\Psi(B, 0)$.

The functions $\Phi(A, \sigma), \Psi(B, \sigma)$ for $\sigma>0$ are then obtained by solving the telegraph equations (28) with these previously determined initial values. For example, using Fourier transforms we obtain the formal solution

$$
\Phi(A, \sigma)=\frac{1}{2 \pi} \iint_{-\infty}^{\infty} \Phi\left(A^{\prime}, 0\right) \exp \left[i k\left(A-A^{\prime}\right)+\lambda^{2} \sigma / 2 i k\right] d A^{\prime} d k .
$$

The lowest-order solution is then given by (10) where $g$ and $h$ are determined by (13), (14), (29) and a corresponding expression for $\Psi(A, \sigma)$. This solution is valid with small error for $t=O\left(\varepsilon^{-2}\right)$.

As remarked in the introduction, for $t=O\left(\varepsilon^{-1}\right)$ a more accurate approximation is obtained by including $\varepsilon u_{1}$ in the solution and this can be obtained from (8), where from (27) we have

$$
G_{1}=-\frac{1}{2} \Phi_{1} \Phi e^{-2 \lambda \tau}+\frac{3}{4 \lambda} \Phi \overline{\Psi^{2}} e^{-3 \lambda \tau}+e^{-\lambda \tau} \tilde{\Phi}(A, \sigma)
$$

with a corresponding expression for $H_{1}$.

The analysis in the paper has applied to a region of infinite spatial extent. As discussed elsewhere [5], such analyses can be extended to bounded regions $(0<x<l)$ with 
fixed-end type boundary conditions (i.e. $u(0, t)=u(l, t)=0$ by the method of odd extension. In the present case, odd extension of $u(x, t)$ about $x=0$ and $x=l$ can be achieved by taking $\Phi(\theta, \sigma)=\Psi(\theta, \sigma)$ for all pairs of arguments $(\theta, \sigma), \Phi(\theta, \sigma)=\Psi(\theta, \sigma)$, and all these functions to be $2 l$-periodic in their first arguments.

With these additions, the foregoing results apply directly to the bounded region problem. It is more appropriate in this case, however, to use a Fourier series solution for $\Phi(A, \sigma)$, rather than (29).

A further point of interest concerns the existence of unidirectional wave solutions. Eqs. (7) for $G$ and $H$ are completely decoupled so that in lowest order the two waves do not interfere with one another. In particular, a unidirectional wave ( $G$ or $H$ zero) can exist. The second-order waves are in general coupled (cf. Eqs. (18) and (19)). However, the second of these equations is identically satisfied by $H=H_{1}=0$, so that for example a unidirectional $G, G$-wave can still exist. (We must have $\bar{\Phi}_{1}=0$ in such a case.) In addition, the interference term in (8) disappears in such a case, and $u$ has the form $u \approx G+\varepsilon G_{1}$ which is purely unidirectional. Furthermore, an examination of the solution (16) for $u_{2}$ reveals that when $H=H_{1}=0, u_{2}$ also has the simple unidirectional form $u_{2}=G_{2}(\alpha, \tau, \sigma)$.

Now let us turn to the case when $\lambda$ is below the critical level and so shocks occur. The foregoing solution can be extended to include shocks provided we add to the basic equation (1) an appropriate jump condition which will enable us to calculate the location of the shock.

We note first that if a shock is fitted into the lowest order solution, $g(\alpha, \tau, 0)$, then equation (15) does give a unique $\alpha$ for each $A$ even if $\lambda$ is below the critical level. The shock provides a dividing line through the region of overlapping characteristics and determines a unique characteristic through each point $(\alpha, \tau)$, and in particular through each point $(A, \infty)$ (see Fig. 1). Thus for each $A$ an initial signal $g\left(\alpha_{1}, 0,0\right)$ is uniquely determined corresponding to the characteristic on which $\alpha \rightarrow A$ as $\tau \rightarrow \infty$.

Consequently $\Phi(A, 0)$ is well defined for all $A$. Then Eq. (28) (through (29), for example) determines $\Phi(A, \sigma)$ for $\sigma>0$. A similar construction provides $\Psi(B, \sigma)$ and then Eq. (30) supplemented by an initial condition for $\tilde{\Phi}$ enables $G_{1}$ and similarly $H_{1}$ to be found. Thus the solution is completely determined once the shock has been fitted.

In order to derive the jump condition we must write Eq. (1) in integrated form. As remarked by Whitham [6], the integrated form is not uniquely determined by the partial differential equation, but we shall assume that it is as follows:

$$
\frac{d}{d t} \int_{x_{1}}^{x_{2}}\left(u_{t}+2 \varepsilon \lambda u\right) d x=\left[u_{x}+u_{x}^{2}\right]_{x_{1}}^{x_{2}} .
$$

This is the simplest integrated form we could take for Eq. (1), and moreover it has the natural form for a momentum equation in cases where $u(x, t)$ is a displacement.

If a shock occurs at $x=s(t)$ then we allow $x_{1} \rightarrow s-0$ and $x_{2} \rightarrow s+0$ in (31), thus obtaining the jump condition

$$
-\dot{s}\left[u_{t}+2 \varepsilon \lambda u\right]=\left[u_{x}+\varepsilon u_{x}^{2}\right]
$$

where square brackets are used as usual to denote the discontinuity of the quantity enclosed within them. Since $u$ is continuous, this simplifies to:

$$
-\dot{s}\left[u_{t}\right]=\left[u_{x}+\varepsilon u_{x}^{2}\right] .
$$


We shall restrict attention in what follows to unidirectional waves, taking $H=H_{1}=$ $H_{2}=0$. Then from Eqs. (6), (8) and (16) we obtain simply that

$$
u=G(\alpha, \tau, \sigma)+\varepsilon G_{1}(\alpha, \tau, \phi)+\varepsilon^{2} G_{2}(\alpha, \tau, \sigma)
$$

and therefore

$$
\begin{aligned}
{\left[u_{t}\right] } & =\left[g+\varepsilon\left(G_{\tau}+g_{1}\right)+\varepsilon^{2}\left(G_{\sigma}+G_{1 \tau}+g_{2}\right)\right] \\
& =\left[g+\varepsilon\left(\frac{1}{2} g^{2}+g_{1}\right)+\varepsilon^{2}\left(g g_{1}+g_{2}\right)\right]
\end{aligned}
$$

and

$$
\left[u_{x}+\varepsilon u_{x}^{2}\right]=\left[g+\varepsilon\left(g_{1}+g^{2}\right)+\varepsilon^{2}\left(g_{2}+2 g g_{1}\right)\right] .
$$

In obtaining the first of these equations we have used Eqs. (7) and (18) and discarded terms which are continuous.

Suppose that the shock occurs at the position $\alpha=\alpha_{s}(\tau, \sigma)$, i.e. at $t+x=\alpha_{s}(\tau, \sigma)$. Then $s(t)=-t+\alpha_{s}(\tau, \sigma)$ and so Eq. (32) becomes

$$
\left(1-\varepsilon \alpha_{s \tau}-\varepsilon^{2} \alpha_{s \sigma}\right)\left[g+\varepsilon\left(\frac{1}{2} g^{2}+g_{1}\right)+\varepsilon^{2}\left(g g_{1}+g_{2}\right)\right]=\left[g+\varepsilon\left(g_{1}+g^{2}\right)+\varepsilon^{2}\left(g_{2}+2 g g_{1}\right)\right] \text {. }
$$

After simplification this becomes

$$
\alpha_{s \tau}\left[g+\varepsilon\left(\frac{1}{2} g^{2}+g_{1}\right)\right]+\varepsilon \alpha_{s \sigma}[g]=-\frac{1}{2}\left[g^{2}\right]-\varepsilon\left[g g_{1}\right] .
$$

Eq. (33) provides us with the required equation for the shock position $\alpha_{s}$ in terms of the solutions $g$ and $g_{1}$ on either side of the shock. In lowest order it reduces to the familiar expression

$$
\alpha_{s t}=-\frac{1}{2}\left[g^{2}\right] /[g]=-\frac{1}{2}\left(g_{+}+g_{-}\right) .
$$

Thus, in principle at least, we are in a position to fit the shock and hence complete the solution.

\section{REFERENCES}

[1] R. W. Lardner, The formation of shock waves in KBM solutions of hyperbolic differential equations, J. Sound \& Vibr. 39, 489-502 (1975)

[2] A. H. Nayfeh, Finite-amplitude longitudinal waves in non-uniform bars, J. Sound \& Vibr. 42, 357-381 (1975)

[3] S. C. Chikwendu and J. Kevorkian, A perturbation method for hyperbolic equations with small nonlinearities, SIAM J. Appl. Math. 22, 235-258 (1972)

[4] A. H. Nayfeh, Perturbation methods, chap. vi (John Wiley, 1973)

[5] R. W. Lardner, Asymptotic solutions of nonlinear wave equations using the methods of averaging and twotiming, Quart. Appl. Math. 35, 225-238 (1977)

[6] G. B. Whitham, Linear and nonlinear waves, Wiley-Interscience, 1974, Sec. 2.7 